Internist $2015 \cdot 56: 473-474$

DOI 10.1007/s00108-014-3625-4

Online publiziert: 19. April 2015

(c) Springer-Verlag Berlin Heidelberg 2015

M. Reincke ${ }^{1} \cdot H$. Lehnert $^{2}$

${ }^{1}$ Medizinische Klinik Innenstadt, Klinikum der Universität München

2 Medizinische Klinik I, Universitätsklinikum Schleswig-Holstein, Campus Lübeck

\title{
Diabetes und seine Komplikationen
}

\section{Diagnostik und Therapie einer Volkskrankheit}

Der Begriff des Kardiologen, Gynäkologen oder auch Urologen ist den meisten Menschen in Deutschland gut bekannt. Deutlich weniger geläufig sind unverändert der Begriff des Endokrinologen und Diabetologen und die damit verbundenen Tätigkeitsfelder. Ebenso unverändert stellt sich die Frage, worin dies begründet ist. Sicher nicht darin, dass nur wenige in Deutschland an den entsprechenden Hormon- und Stoffwechselstörungen leiden.

Die aktuellen Zahlen sprechen nämlich eine andere Sprache: In Deutschland sind Millionen von Menschen von Störungen der inneren Sekretion betroffen: Diabetes (7 Mio. Betroffene), metabolisches Syndrom und schweres Übergewicht (16 Mio. Betroffene), Struma und andere Schilddrüsenerkrankungen (etwa $30 \%$ der Bevölkerung), Osteoporose, ungewollte Kinderlosigkeit bei einem Syndrom der polyzystischen Ovarien (etwa 1 Mio.) gehören zu den Volkskrankheiten.

Der Diabetes liegt nach Angaben der Weltgesundheitsorganisation inzwischen auf Platz 4 der Todesursachen weltweit (www.who.int/mediacentre/factsheets/ fs355/en/) und betrifft in Deutschland rund 9\% der erwachsenen Bevölkerung. Nach den Zahlen des Deutschen Gesundheitssurvey aus dem Jahr 2012 haben in Deutschland 7,2\% der Bevölkerung einen bekannten Diabetes und zusätzlich 2,1\% einen unentdeckten Diabetes (insgesamt 9,3\%). Diese Zahl basiert auf einer repräsentativen nationalen Bevölkerungsstichprobe im Alter von 18-79 Jahren (http:// www.diabetes-deutschland.de/aktuellesituation.html).

Die deutsche Bevölkerung weist dabei erhebliche regionale Unterschiede in der Krankheitslast metabolischer Erkrankungen auf [1]. Die standardisierte Prävalenz des Diabetes lag in den östlichen Bundesländern mit 12,0\% (10,3-13,7\%) deutlich höher als im Süden mit 5,8\% (4,9$6,7 \%)$. Gleichzeitig erfolgt die Behandlung mit oralen Antidiabetika häufiger im Süden (57\%) als im Nordosten (46\%), während eine Insulinmonotherapie häufiger im Nordosten (22\%) als im Süden (16\%) durchgeführt wird.

》) Der Diabetes liegt weltweit auf Platz 4 der Todesursachen

Ganz aktuelle Zahlen zur Entwicklung der Adipositas- und Diabetesprävalenz stammen aus der Study of Health in Pomerania (SHIP; - Abb. 1). Im Vergleich zu einer ersten Querschnittstudie von 1997 bis 2001 (SHIP-0) mit 4308 Teilnehmern zeigte sich in der Nachfolgestudie SHIPTrend mit 4420 Teilnehmern, durchgeführt zwischen 2008 und 2012, eine starke Zunahme des Body-Mass-Index von 26,7 auf $27,5 \mathrm{~kg} / \mathrm{m}^{2}$ und gleichzeitig eine $\mathrm{Zu}$ nahme der Adipositasprävalenz von $25 \%$ auf $32 \%$ [2]. Parallel wurde in dieser Kohorte eine Zunahme der Diabetesprävalenz von $9,1 \%$ auf $13,8 \%$ beobachtet.

Diese drastische Zunahme der Adipositas- und Diabetesprävalenz deutet auf ein weiter ansteigendes metabolisches
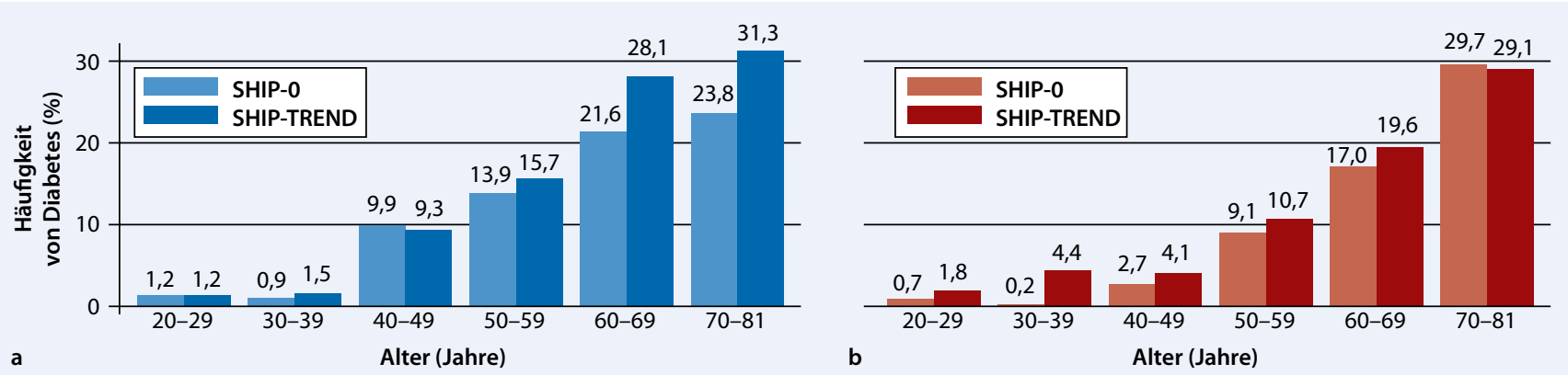

Abb. 1 D Diabetesprävalenz von 1997 bis 2001 (SHIP-0) im Vergleich zum Zeitraum von 2008 bis 2012 (SHIP-TREND) bei a Männern und $\mathbf{b}$ Frauen. (Adaptiert nach [2]) 
Risikoprofil in der deutschen Bevölkerung hin. Daher bedarf es einer konzertierten Aktion unseres Gesundheitswesens, um diesem Trend entgegenzuwirken. Die Deutsche Gesellschaft für Endokrinologie (DGE) hat nun eine Informationskampagne unter dem Schlagwort „Hormongesteuert?!“- Warum Endokrinologie für jeden wichtig ist gestartet (www. endokrinologie.net/presse_150318.php). Hierdurch soll das Bewusstsein für metabolische Erkrankungen geschärft und damit ein wesentlicher Beitrag zur Aufklärung und damit Vorbeugung, Früherkennung und fachgerechten Behandlung geleistet werden.

Die sechs Schwerpunktbeiträge der vorliegenden Ausgabe von Der Internist sollen den aktuellen Informationsstand zur Behandlung des Diabetes, aber auch seiner Komplikationen vertiefen. Neue Optionen für das diagnostische und therapeutische Management werden aufgezeigt.

Die Arbeit von M. Hummel u. P. Achenbach zeigt sehr deutlich, dass die Inzidenz des Typ-1-Diabetes weltweit deutlich ansteigt. Hieraus leiten die Autoren die unbedingte Notwendigkeit von Früherkennung und präventiven Strategien ab. Das Risiko der Entwicklung eines Typ-1-Diabetes kann heute sehr genau definiert werden, sodass insbesondere auch eine Primärprävention ein bedeutsamer Ansatz ist. Der Beitrag von G. Freckmann zu neuen Technologien in der Diabetologie beschreibt die Entwicklung und den Einsatz insbesondere von neuen Blutzuckermesssystemen, die eine kontinuierliche Messung und damit eine wesentlich bessere und v. a. alltagsnähere Blutzuckereinstellung erlauben. Ein Beispiel ist das minimal-invasive Nadelsensorsystem. Auch wenn wir noch ein Stück vom „closed loop“ entfernt sind, zeigen erste Modelle vielversprechende Ergebnisse, die zumindest den Einsatz von teilautomatisierten Systemen in näherer Zukunft erwarten lassen. Die Therapie des Typ-2-Diabetes mit medikamentösen Maßnahmen und einer Insulinbehandlung wird von E. Siegel dargestellt; sein Beitrag verdeutlicht sehr eindrucksvoll, dass auch der Typ-2-Diabetes eine sehr heterogene Erkrankung ist, für deren Behandlung heute unterschiedliche medikamentöse Strategien verfügbar sind. Von großer klini- scher Bedeutung ist der Hinweis auf ein wesentliches Therapieziel: Das Hypoglykämierisiko muss minimiert und eine Gewichtszunahme vermieden werden. Der Weg hin zu einem individuellen $\mathrm{HbA}_{1 \mathrm{C}^{-}}$ Ziel unter Berücksichtigung der individuellen Eigenschaften des Patienten war und ist sicher einer der großen konzeptuellen Fortschritte in der Diabetestherapie.

\section{》) Die Rate diabetesbedingter Amputationen ist in Deutschland weiterhin viel zu hoch}

Neuropathie und diabetisches Fußsyndrom stellen unverändert nicht nur hochrelevante Komplikationen des Diabetes dar, sondern insbesondere auch eine sehr komplexe Herausforderung an den behandelnden Arzt. R. Lobmann beschreibt umfassend die Möglichkeiten der Primärund Sekundärprävention - immer mit dem Ziel, die weiterhin viel zu hohe Amputationsrate in Deutschland signifikant zu senken. Unverändert ist eine der häufigsten Ursachen der chronischen Niereninsuffizienz die diabetische Nephropathie, die von S. Werth, H. Lehnert und J. Steinhoff diskutiert wird. Auch hier bietet die frühzeitige Detektion einer Mikroalbuminurie die Möglichkeit zu wirksamen präventiven Strategien. Beachtet werden muss, dass die Albuminurie selbst ein genuiner kardiovaskulärer Risikofaktor ist, darüber hinaus natürlich auch ein Surrogatmarker für weitere Endothelschäden. Die Blutdruckzielwerte werden anhand der neuen Leitlinien diskutiert; unverändert gelten hier Medikamente mit dem Ziel der RAAS-Blockade als Therapie der Wahl, allerdings nicht in Kombination. Neue Pharmaka, insbesondere zur Modulation inflammatorischer Signalwege, werden derzeit erprobt. D. Kopf berichtet schließlich in seinem Beitrag über den Zusammenhang zwischen Diabetes mellitus und Demenz. Der Diabetes gilt inzwischen als etablierter Risikofaktor für eine Demenzerkrankung - die Mechanismen sind komplex und beruhen auf vaskulären und entzündlichen Ursachen, einer Akkumulation toxischer Stoffwechselprodukte, aber auch auf häufigen und schweren Hypoglykämien. Gerade weil auch das Lebensalter diabetischer Patienten zunimmt, ist die Früherkennung, Prävention und Behandlung demenzieller Probleme von größter Bedeutung.

Die Herausgeber danken allen Autoren herzlichst für ihre wichtigen und spannenden Beiträge und hoffen sehr, mit dieser Ausgabe von Der Internist zu einer noch besseren Wahrnehmung des Problems Diabetes und seiner möglichst frühen Diagnostik und Behandlung beizutragen.

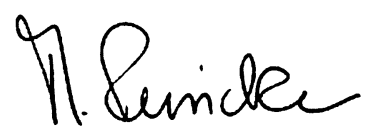

Martin Reincke

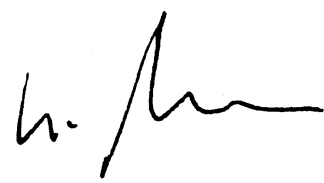

Hendrik Lehnert
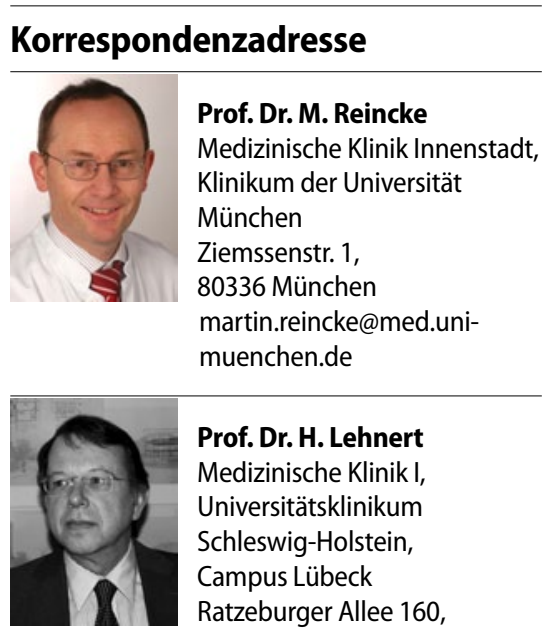

Prof. Dr. H. Lehnert Medizinische Klinik I, Universitätsklinikum Schleswig-Holstein, Campus Lübeck Ratzeburger Allee 160, 23562 Lübeck hendrik.lehnert@uk-sh.de

Interessenkonflikt. M. Reincke und H. Lehnert geben an, dass kein Interessenkonflikt besteht.

\section{Literatur}

1. Schipf S, Werner A, Tamayo T et al (2012) Regional differences in the prevalence of known type 2 diabetes mellitus in 45-74 years old individuals: results from six population-based studies in Germany (DIAB-CORE Consortium). Diabet Med 29:e8895

2. Völzke H, Ittermann T, Schmidt CO et al (2015) Prevalence trends in lifestyle-related risk factors - two cross-sectional analyses with a total of 8728 participants from the Study of Health in Pomerania from 1997 to 2001 and 2008 to 2012. Dtsch Arztebl Int 112:185-192 